\title{
Development of the Baldin approach for the relativistic nuclear interactions
}

\author{
Denis Artemenkov ${ }^{1, \star}$, Alexander Malakhov ${ }^{1, \star \star}$, and Gennady Lykasov ${ }^{1, \star \star \star}$ \\ ${ }^{1}$ Joint Institute for nuclear research, Dubna, Moscow region, 141980, Russia
}

\begin{abstract}
This article presents the development of the A.M.Baldin approach using a description of relativistic nuclear interactions in the four velocity space. The modification of this approach taking into account the energy dependence of inclusive spectra of hadron has allowed us to describe the differential cross section as a function of the transverse momentum and a satisfactory description of the slopes of the hadron spectra as functions of the energy, has been obtained. The hadron spectra as functions of the energy, has been obtained. Results of our calculations have coincided with experimental data in a wide energy range with high precision.
\end{abstract}

\section{Introduction}

A.M. Baldin was invited to consider relativistic nuclear interactions in the four dimension velocity space. The approach of studying relativistic nuclear interactions in the four dimension velocity space proved to be very fruitful. This approach allowed him to classify nuclear interactions, effectively highlight the four-dimensional hadron jets, and study the asymptotic properties of the nuclear interactions at high energies [1]-[3]. This article presents further development of this approach.

Let us put down the interaction of nucleus $I$ with nucleus II resulting in the inclusive particle 1 production as follows:

$$
I+I I \rightarrow 1+\ldots
$$

According to Baldin's assumption more than one nucleon in the nucleus $I$ can participate in the interaction (1). The value of $N_{I}=\lambda \cdot A_{I}$ is the efficient number of nucleons inside the nucleus $I$, participating in the interaction which is called the cumulative number. Its values lie in the region of $0 \leq N_{I} \leq A_{I}$. The cumulative area complies with $N_{I}>1$. Of course, the same situation will be for the nucleus II, and you can enter the cumulative number of $N_{I I}$.

For reaction (1) with the production of the inclusive particle 1, we can write the law of conservation of four-momentum in the following form:

$$
\left(N_{I} P_{I}+N_{I I} P_{I I}-p_{1}\right)^{2}=\left(N_{I} m_{0}+N_{I I} m_{0}+M\right)^{2},
$$

where $N_{I}$ and $N_{I I}$ are cumulative numbers (the number of nucleons involved in the interaction); $P_{I}$, $P_{I I}, p_{1}$ are four-momenta of the nuclei $I$ and II and particle 1 , respectively; $m_{0}$ is the mass of the

\footnotetext{
^e-mail: artemenkov@1he.jinr.ru

$\star \star$ e-mail: malakhov@lhe.jinr.ru

$\star \star \star$ e-mail: lykasov@jinr.ru
} 
nucleon; $M$ is the mass of the particle providing the conservation of the baryon number, strangeness, and other quantum numbers. For particles produced without accompanying antiparticles ( $\pi$ mesons, jets and others) $M=0$. In [3] the parameter of self-similarity was introduced, that allowed one to describe the differential cross section of the yield of a large class of particles in relativistic nuclear collisions:

$$
\Pi=\min 1 / 2\left[\left(u_{I} N_{I}+u_{I I} N_{I I}\right)^{2}\right]^{1 / 2},
$$

where $u_{I}$ and $u_{I I}$ are four-velocities of the nuclei $I$ and $I I$.

In this case the invariant cross-sections of the output inclusive particles of different types at nucleus interactions with atomic numbers $A_{I}$ and $A_{I I}$, are described by the universal dependence in a wide energy range and different atomic numbers of the colliding nuclei:

$$
E \cdot d^{3} \sigma / d p^{3}=C_{1} A_{I}^{\alpha\left(N_{I}\right)} \cdot A_{I I}^{\alpha\left(N_{I I}\right)} \cdot \exp \left(-\Pi / C_{2}\right)
$$

where $\alpha\left(N_{I}\right)=1 / 3+N_{I} / 3, \alpha\left(N_{I I}\right)=1 / 3+N_{I I} / 3, C_{1}=1.9 \cdot 10^{4} \mathrm{mb} \cdot \mathrm{GeV}^{-2} \cdot c^{3} \cdot \mathrm{st}^{-1}$ and $C_{2}=$ $0.125 \pm 0.002$.

\section{Self-similarity parameter in the central rapidity region}

In the central rapidity region it is possible to find the analytical expression for $\Pi$ [4]. For this case $N_{I}$ and $N_{I I}$ are equal to each other: $N_{I}=N_{I I}=N$.

$$
N=\left[1+\left(1+\Phi_{\delta} / \Phi^{2}\right)^{1 / 2}\right] \Phi
$$

where

$$
\begin{gathered}
\Phi=2 m_{0}\left(m_{1 t} \cosh Y+M\right) / \sinh ^{2} Y, \\
\Phi_{\delta}=\left(M^{2}-m_{1}^{2}\right) /\left(4 m_{0}^{2} \cdot \sinh ^{2} Y\right) .
\end{gathered}
$$

Here $m_{1 t}=\left(m_{1}^{2}+p_{t}^{2}\right)^{1 / 2}$ is the transverse mass of the particle $1, Y$ is the rapidity of the colliding nuclei. And then

$$
\Pi=N \cosh Y
$$

\section{Further development of Baldin's approach}

For the inclusive cross-section of hadron production in the central rapidity region and according to AGK (Abramovsky, Gribov, Kanchelly) cancellation [5] we can write the following expression for the quark contribution:

$$
E\left(d^{3} \sigma / d p^{3}\right)_{q}=\phi_{q}\left(y=0, p_{t}\right) \cdot \Sigma\left[n \sigma_{n}(s)\right]
$$

According to [6] we write:

$$
E\left(d^{3} \sigma / d p^{3}\right)_{q}=\phi_{q}\left(y=0, p_{t}\right) \cdot \Sigma\left[n \sigma_{n}(s)\right]=\phi_{q}\left(y=0, p_{t}\right) \cdot g \cdot\left(s / s_{0}\right)^{\Delta}
$$

According to [7], [8] the inclusive cross-section of hadron production, taking into account the gluon contribution, can be written in the following form:

$$
\begin{gathered}
E\left(d^{3} \sigma / d p^{3}\right)_{g}=\phi_{g}\left(y=0, p_{t}\right) \cdot \Sigma\left[n \sigma_{n}(s)\right]=\phi_{g}\left(y=0, p_{t}\right) \cdot \Sigma\left[(n-1) \cdot \sigma_{n}(s)\right]= \\
\phi_{g}\left(y=0, p_{t}\right) \cdot\left[g \cdot\left(s / s_{0}\right)^{\Delta}-\sigma_{n d}\right] .
\end{gathered}
$$




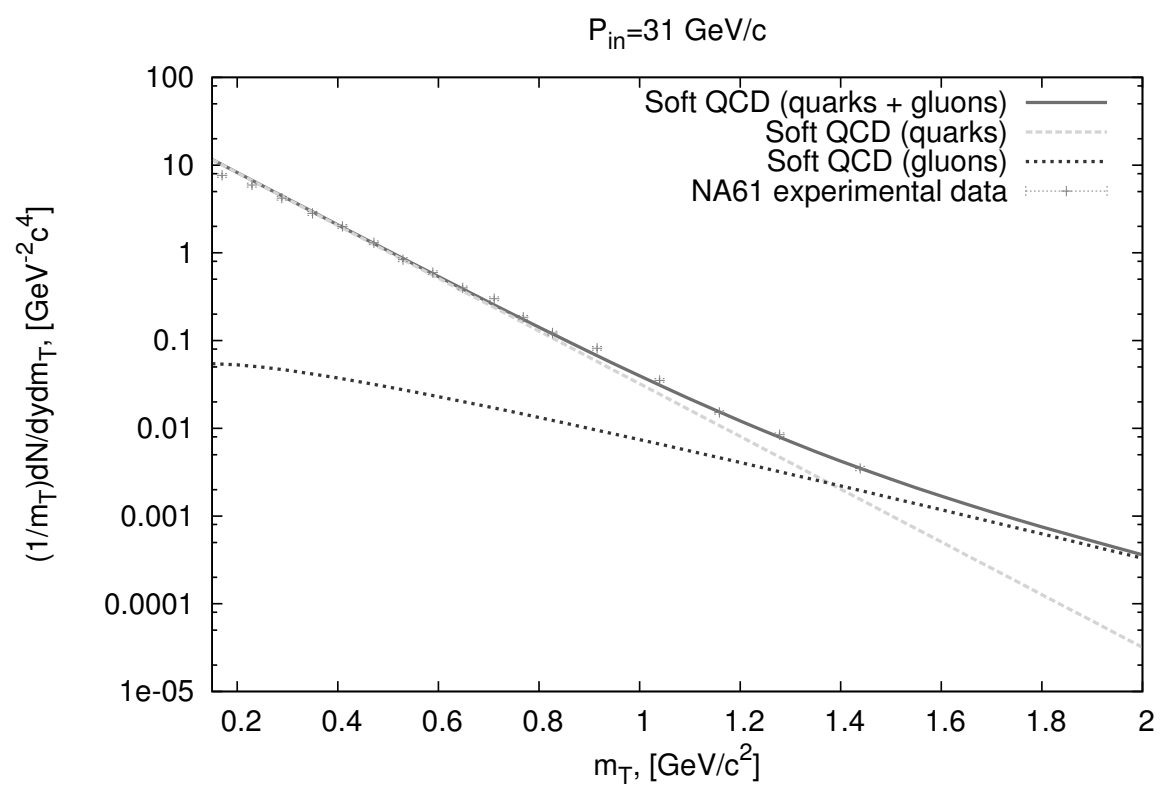

Figure 1. Results of the calculations of the inclusive cross-section of pion production in pp collisions as a function of the transverse mass at the initial momenta $P_{i n}=31 \mathrm{GeV} / c$. They are compared to the NA61 experimental data [9].

Thus, taking into account the quark and gluon contributions we will obtain the following expression:

$$
E\left(d^{3} \sigma / d p^{3}\right)=\left[\phi_{q}\left(y=0, p_{t}\right)+\phi_{g}\left(y=0, p_{t}\right) \cdot\left(1-\sigma_{n d} /\left[g\left(s / s_{0}\right)^{\Delta}\right)\right] \cdot g \cdot\left(s / s_{0}\right)^{\Delta} .\right.
$$

In formulas (9)-(12) we apply the following symbols: $\sigma_{n}$ - cross-section of hadron production by means of the n-pomeron exchange; $\phi=\phi(\Pi) ; g$ - constant ( $\sim 20$ mbarn $)$, which is calculated within the "quasi-eikonal" approximation [6]; $S_{0}=1 \mathrm{GeV}^{2} ; \Delta=\left[\alpha_{p}(0)-1\right] \sim 0.08$, where $\alpha_{p}(0)$ is the subcritical Pomeron intercept.

The first part of the inclusive spectrum (Soft QCD (quarks)) is related to the function $\phi_{q}(y=0, \Pi)$, which is fitted by the following form:

$$
\phi_{q}(y=0, \Pi)=A_{q} \exp \left(-\Pi / C_{q}\right),
$$

where $A_{q}=3.68(\mathrm{GeV} / c)^{-2}, C_{q}=0.147$.

The function $\phi_{q}(y=0, \Pi)$ related to the second part (Soft QCD (gluons)) of the spectrum is fitted by the following form:

$$
\phi_{g}(y=0, \Pi)=A_{g} \sqrt{m_{1 t}} \exp \left(-\Pi / C_{g}\right),
$$

where $A_{g}=1.7249(\mathrm{GeV} / c)^{-2}, C_{g}=0.289$. Using (12) we can calculate the inclusive cross section of hadron production as a function of the transverse mass.

In figure 1 the inclusive spectrum $\left(1 / m_{1 t}\right) d \sigma / d m_{1 t} d y$ of $\pi^{-}$-mesons produced in pp collisions at the initial momentum $P_{\text {in }}=31 \mathrm{GeV} / c$ is presented versus their transverse mass $m_{t}$. Using only the first part of the spectrum $P_{\text {in }}=158 \mathrm{GeV} / c$, which is due to the quark contribution, the conventional string 


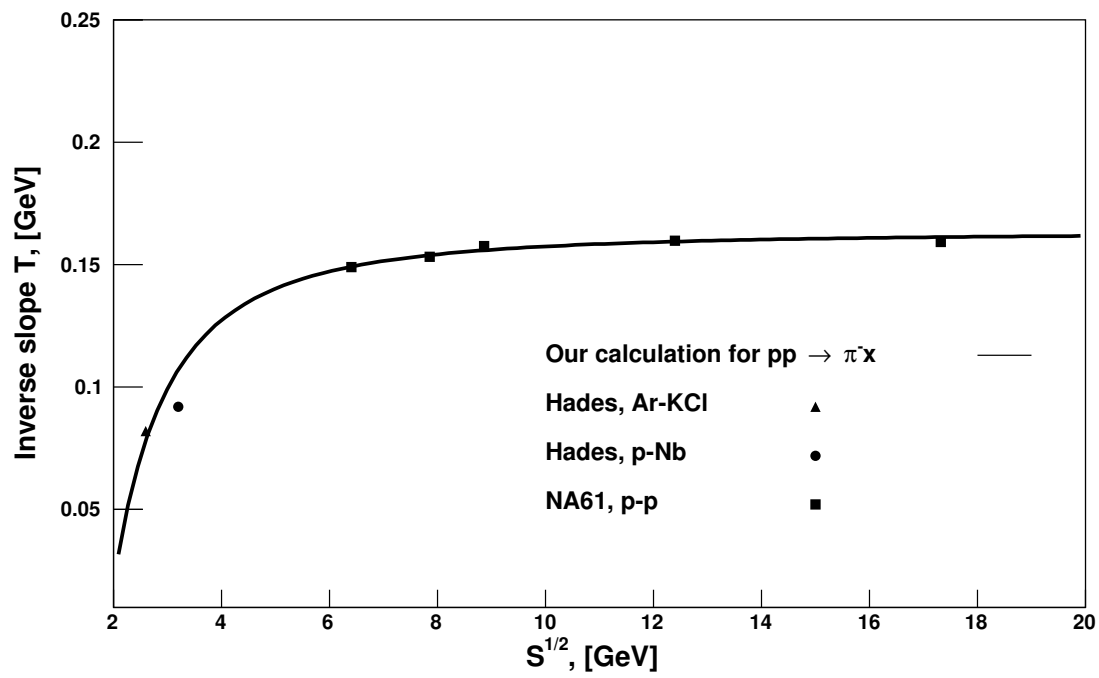

Figure 2. Results of calculations of the inverse slope parameter $T$ on the energy dependence for the negative pion production in $\mathrm{pp}$ interactions. The experimental points are taken from $[9,10]$.

model, let us call it the Soft QCD (quarks), one can describe the NA61 data [9] rather satisfactorily at $p_{\text {in }}=31 \mathrm{GeV} / c$ and $m_{1 t}<1 \mathrm{GeV} / c^{2}$.

This part of the inclusive spectrum corresponds to the dashed line in figure 1. The inclusion of the second part of spectrum due to the contribution of gluons (Soft QCD (gluons)), the dotted line, allowed us to describe all the NA61 data up to $m_{1 t}=1.5 \mathrm{GeV} / c$, see the solid line in figure 1 (Soft QCD (quarks + gluons)).

Figure 1 shows that the deviation of the simple exponential form of the pion spectrum (dashed line) from the total description of the NA61 data (solid line) appears only at $m_{1 t}>0.8 \mathrm{GeV} / c^{2}$ and it is very small. Therefore, the spectrum approximately can be presented in the exponential form, which depends both on $m_{1 t}$ and $s$ [13]:

$$
\begin{gathered}
\rho\left(s, m_{1 t}\right) \simeq \phi_{q}(y=0, \Pi) g\left(s / s_{0}\right)^{\Delta}=g\left(s / s_{0}\right)^{\Delta} A_{q} \exp \left(-\frac{m_{1 t}}{C_{q}^{\prime} m_{0}\left(1-4 m_{0}^{2} / s\right)}\right)= \\
g\left(s / s_{0}\right)^{\Delta} A_{q} \exp \left(-m_{1 t} / T\right),
\end{gathered}
$$

were

$$
T=C_{q}^{\prime} m_{0}\left(1-4 m_{0}^{2} / s\right)
$$

is the inverse slope parameter, which is called sometimes as the thermal freeze-out temperature.

Thus the value of $T$ given by (16) is in direct relation of the similarity parameter of $\Pi$ to the Mandelstam relativistic invariant variables $s, t, u$.

One can see from (16) that this thermal freeze-out temperature depends on the initial energy of collided protons. That is the direct consequence of the self-similarity approach, which operates the four-momentum velocity formalism. This $s$-dependence of $T$ is significant at low initial energies and at $s \gg m_{0}^{2}$ the slope parameter $T$ becomes independent of $s$. We have calculated the inverse slope parameter $T$, as a function of the energy $\sqrt{s}$ given by (16) and presented in figure 2 . There is a good agreement with the experimental data [9]. 
In figure 3 we present the calculations of inclusive spectra of charged hadrons (mainly pions and kaons) produced in pp collision at $\sqrt{s}=7 \mathrm{TeV}$ performed by using (12) and the perturbative QCD (PQCD) within the leading order (LO) $[7,8]$ compared to the LHC data.

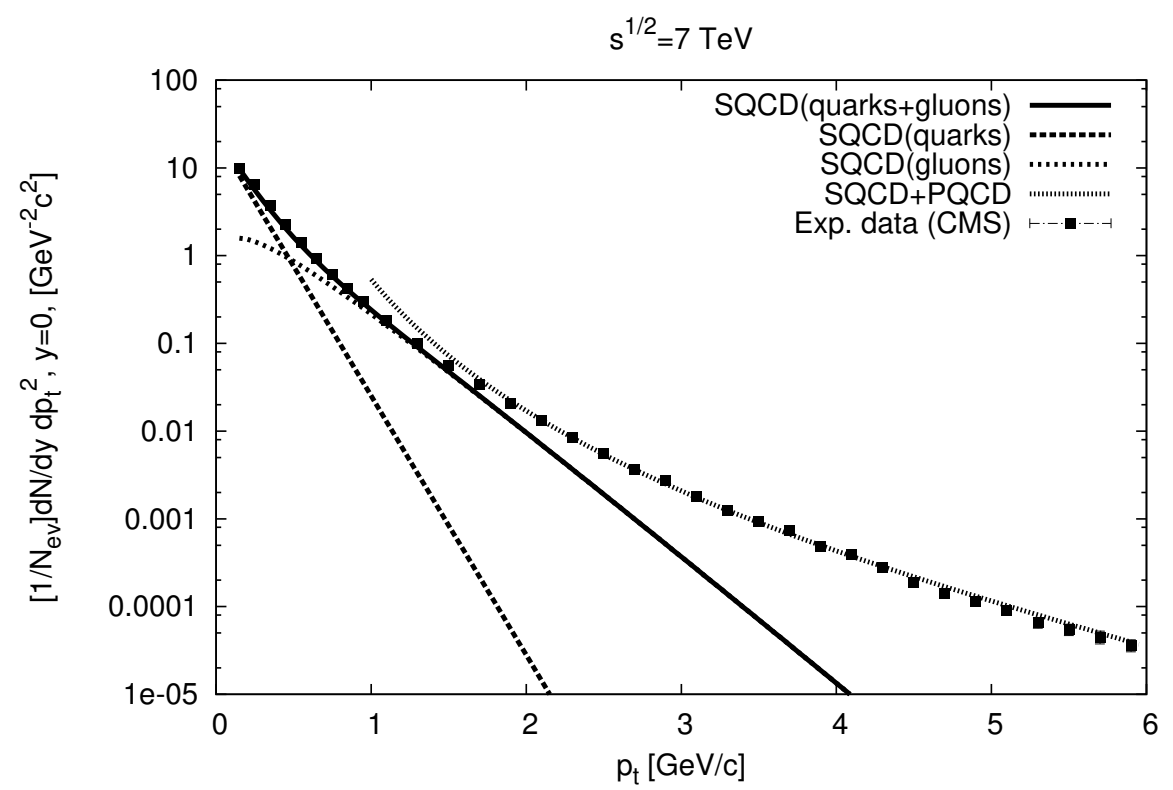

Figure 3. Results of the calculations of the inclusive cross-section of charge hadrons produced in pp collisions at the LHC energies as a function of their transverse momentum pt at $\sqrt{s}=7 \mathrm{TeV}$. The points are the LHC experimental data $[11,12]$.

These spectra are the sums of inclusive spectra of pions and kaons, therefore they are presented as a function of the transverse momentum $p_{t}$ instead of functions of the transverse mass $m_{1 t}$ because the masses of the pion and kaon are different. In addition to the part of spectrum, which corresponds to (12), see the solid lines in these figures, we have also included the PQCD calculations, see the dotted lines. The PQCD calculation within the LO is divergent at low $p_{t}$, therefore, the dotted lines go up, when $p_{t}$ decreases. The kinematical region about $p_{t} \simeq 1.8-2.2 \mathrm{GeV} / c$ can be treated as the matching region of the nonpertubative QCD (Soft QCD) and the pertubative QCD PQCD. From figure 3, one can see that it is possible to describe rather well these inclusive spectra in a wide region of $p_{t}$ at the LHC energies matching these two approaches. In [13] there are more details of the results of our calculations for the inclusive cross sections of charge hadrons produced in pp collisions at the SPS and LHC energies.

\section{Conclusion}

The inclusive hadron spectrum in the space of four velocities is presented within the self-similarity approach as a function of the similarity parameter $\Pi$.

We have shown that the energy dependence of the similarity parameter $\Pi$ included within this approach is very significant at low energies, namely at $\sqrt{s}<6 \mathrm{GeV}$, and rather well reproduces the experimental data on the inverse slope or the thermal freeze-out temperature of the inclusive 
spectrum of hadrons produced in pp collisions, it increases and saturates when $\sqrt{s}$ grows. This is very significant for a theoretical interpretation of future experimental data planned to obtain at FAIR, CBM (Darmstadt, Germany) and NICA (Dubna, Russia) projects.

However, we have also shown that the $s$-dependence of $\Pi$ is not enough to describe the inclusive spectra of hadrons produced in the midrapidity region, for example, in pp collisions in the wide region of initial energy, especially at the LHC energies. Therefore, we modify the self-similarity approach using the quark gluon string model (QGSM) including the contribution of non-perturbative gluons, which are very significant to describe the experimental data on inclusive hadron spectra in the midrapidity region at the transverse momenta $p_{t}$ up to $2-3 \mathrm{GeV} / c$.

To describe the data in the midrapidity region and values of $p_{t}$ up to $2-3 \mathrm{GeV} / c$, we have modified the simple exponential form of the spectrum, as a function of $\Pi$, and presented it in two parts due to the contribution of quarks and gluons, each of them has different energy dependence. To extend the application of the suggested approach to analyze these inclusive $p_{t}$-spectra at large hadron transverse momenta, we have to include the perturbative QCD (PQCD) calculation, which results in the main contribution at $p_{t}>2-3 \mathrm{GeV} / c$.

\section{References}

[1] A. M. Baldin, L. A. Didenko, Fortsch.Phys. 38, 261-332 (1990)

[2] A. M. Baldin, A. I. Malakhov, and A. N. Sissakian, Physics of Particles and Nuclei Suppl. 32, S4-S30 (2001)

[3] A. M. Baldin, A. A. Baldin, Phys. Particles and Nuclei 29(3), 232 (1998)

[4] A. M. Baldin, A. I. Malakhov, JINR Rapid Communications (87)-98(1), 5-12 (1998)

[5] V. Abramovsky, V. N. Gribov and O. Konchelli, Sov.J.Nucl.Phys. 18, 308 (1973)

[6] K. A. Ter-Martirosyan, Sov.J.Nucl.Phys. 44, 817 (1986)

[7] V. A. Bednyakov, A. A. Grinyuk, G. I. Lykasov, M. Pogosyan, Int.J.Mod.Phys. A 27, 1250042 (2012)

[8] A. A. Grinyuk, G. I. Lykasov, A. V. Lipatov, N. P. Zotov, Phys.Rev. D 87, 074017 (2013)

[9] A. A. Abgrall et al., Eur.Phys.J. C 74, 2794 (2014)

[10] G. Agakishiev et al., arXiv:1512.07070, Eur.Phys.J. A 52, 178 (2016)

[11] ATLAS Collab. (G. Aad et al.), New J.Phys. 13, 053033 (2011)

[12] CMS Collab. (V. Khachatryan et al.), Phys. Rev. Lett. 105, 022002 (2010)

[13] D. A. Artemenkov, G. I. Lykasov and A. I. Malakhov, Int.J.Mod.Phys. A 30, 1550127 (2015) 